\title{
Spectral Imaging Skin Probe for Chronic Wounds Therapy Evaluation
}

\author{
F. Erfurth, B. Nyuyki, A. Scheibe, W.D. Schmidt \\ Gesellschaft zur Förderung von Medizin-, Bio- und Umwelttechnologien e.V. \\ Fachsektion Photonik und Sensorik \\ Jena (Germany)
}

\section{The problem of chronic wounds}

Motivation for the development of a skin imaging probe was the need for an objective assessment of chronic wounds with regard to the healing process. A chronic wound often represents the last stage of progressive tissue destruction [1]. Most often an insufficient supply of the skin with oxygen and nutrients is the cause. A disturbed blood circulation can have diverse causes, such as pressure over a longer period. Prolonged pressure that exceeds that of the capillaries in the skin, leads to an insufficient supply of the tissue with oxygen, nutrients and antibodies and also to an accumulation of toxic substances. Another common cause of chronic wounds is the development of diabetes mellitus, a permanent increase in blood glucose levels. The disease leads to damage of the nerves especially at distal parts, which makes patients less aware of consequential damages. As a result the diabetic foot syndrome possesses a high risk of infection. Basically, each wound can transform into a chronic wound, if external factors interfere with the healing.

In order to treat a chronic wound properly, it is important to know how large and how deep the wound and in which state of healing it is. Therefore, the physician cleans the wound thoroughly. After the subsequent disinfection a wound dressing is applied. Its task is to keep the wound moist, but also to remove excess fluid from the wound. In addition, it protects against infections. Modern wound dressings actively promote healing. So far, to assess the progress of wound healing, the physician evaluated the condition of the cleaned wound in larger time intervals based on several criteria.

The clinical wound score according to Wollina [2] is a standardizable method to assess wound healing on a scale of eight steps (score 0-7). Here, the quantity, color and firmness of the newly formed granulation tissue are taken in to consideration. The assessment of the individual properties is done, however, subjectively. Thus, it is not ensured that after longer periods, the re-evaluation of the wound will follow the same standards. By means of a hyperspectral image recording of the wound region this task can now be objectified.

\section{Innovation of the hyperspectral skin probe}

Remission spectroscopy has been used in different situations in the diagnosis of skin lesions [2]. This non-invasive optical method is based on the introduction of light into the skin and the detection of the reemitted or backscattered portion. In the skin tissue the light interacts with chromophores like hemoglobin or melanin, as well as water that partly absorbs the light. Hence, the re-emitted spectrum is changed and therefore permits the identification of wound tissue due to its wavelength-dependent response upon its illumination with white light (Fig. 1).

However, problems have been encountered with previous point-wise measurement of skin, since with inhomogeneous samples a single point measurement is insufficient and with repeated measurements the exact measuring position can only be poorly recovered.

A solution to these problems in the past, was a successful combination of point-wise spectroscopic measurements with photography. For this purpose, the GMBU e.V. developed a FluorescenceRemission-Imaging-Sensor (FRIS) [3] with a special probe, with makes the collection of remission and fluorescence from the measuring point possible. In addition, a monitor mode allows for the display of a live image of the skin surface. Alternatively a single frame can be stored. The sensor permits a reproducible spectroscopic measurement of the wound.

Hyperspectral image recording goes one step further. Here, the image generated with a single shot consists completely of remission spectra. This increases the information content of the wound pictures dramatically. Thus, the distribution of granulation tissue in the wound can be shown on the basis of its remission spectra from these data. The same goes for any other wound component. 


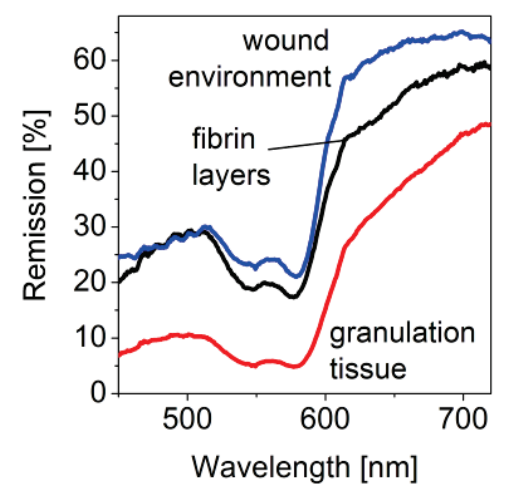

Fig. 1, Remission spectra of components of a chronic wound (in vivo)
Conventional image processing

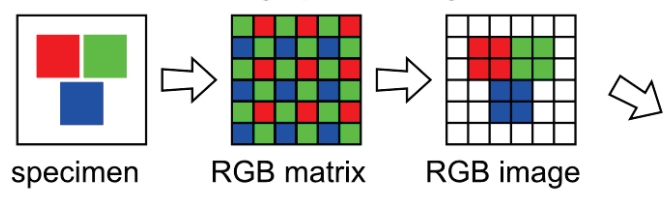

Classical spectroscopy

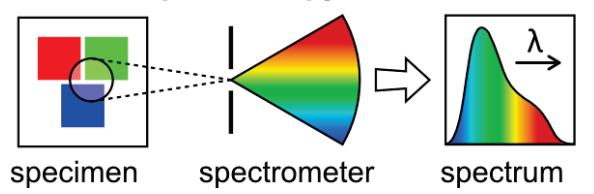

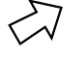

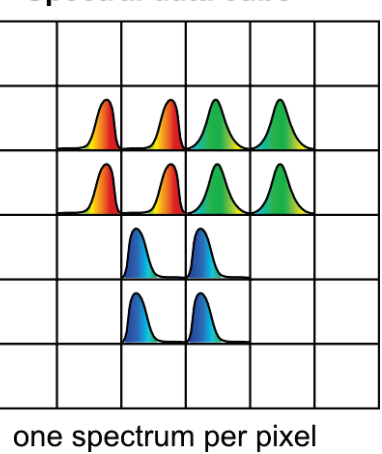

Fig. 2, Spectral Imaging principle: SI combines conventional image processing and classical spectroscopy

\section{Technical implementation of the sensor}

The skin probe is based on the principle of Spectral Imaging (Fig. 2), which represents a combination of classical spectroscopy and digital image processing, whereby a spectrum is recorded for each pixel simultaneously. This is made possible by the use of an optical module, the imaging spectrograph. On the input side, it simultaneously picks up the light from several points arranged along a line through a lens system. Each point on the line is spectrally dispersed in the spectrograph such that at the output each individual point forms a spectrum on the image sensor of the camera. This way, several hundred spectra can be acquired simultaneously.

The GMBU e.V. has already used this technology to develop sensors for other applications, such as hyperspectral image recording of multicolor genotyping microarrays with highly sensitive fluorescence detection [4]. Another example is a device for optical flame analysis and optimization of gas supply for industrial burners [5]. Here the emitted radiation along the gas burner flame is spatially resolved and spectrally evaluated. A third sensor described here as skin probe, represents a variation of the measuring principle used in both cases. However, while in the first two cases, the radiation is gathered from a linear region, the skin probe takes up the emission from a planar surface of $40 \times 40 \mathrm{~mm}^{2}$ simultaneously.

The structure of the skin probe can be described by two optical branches, one for illumination and one for detection (Fig. 3). For illuminating the skin a halogen lamp is used, whose radiation is focused through condenser lens optics. An electro-mechanical shutter, situated before the light source, enables quick reference measurements without the integrated lighting. The light from the halogen lamp is homogenized and coupled into a bundle of 144 individual optical fibers.

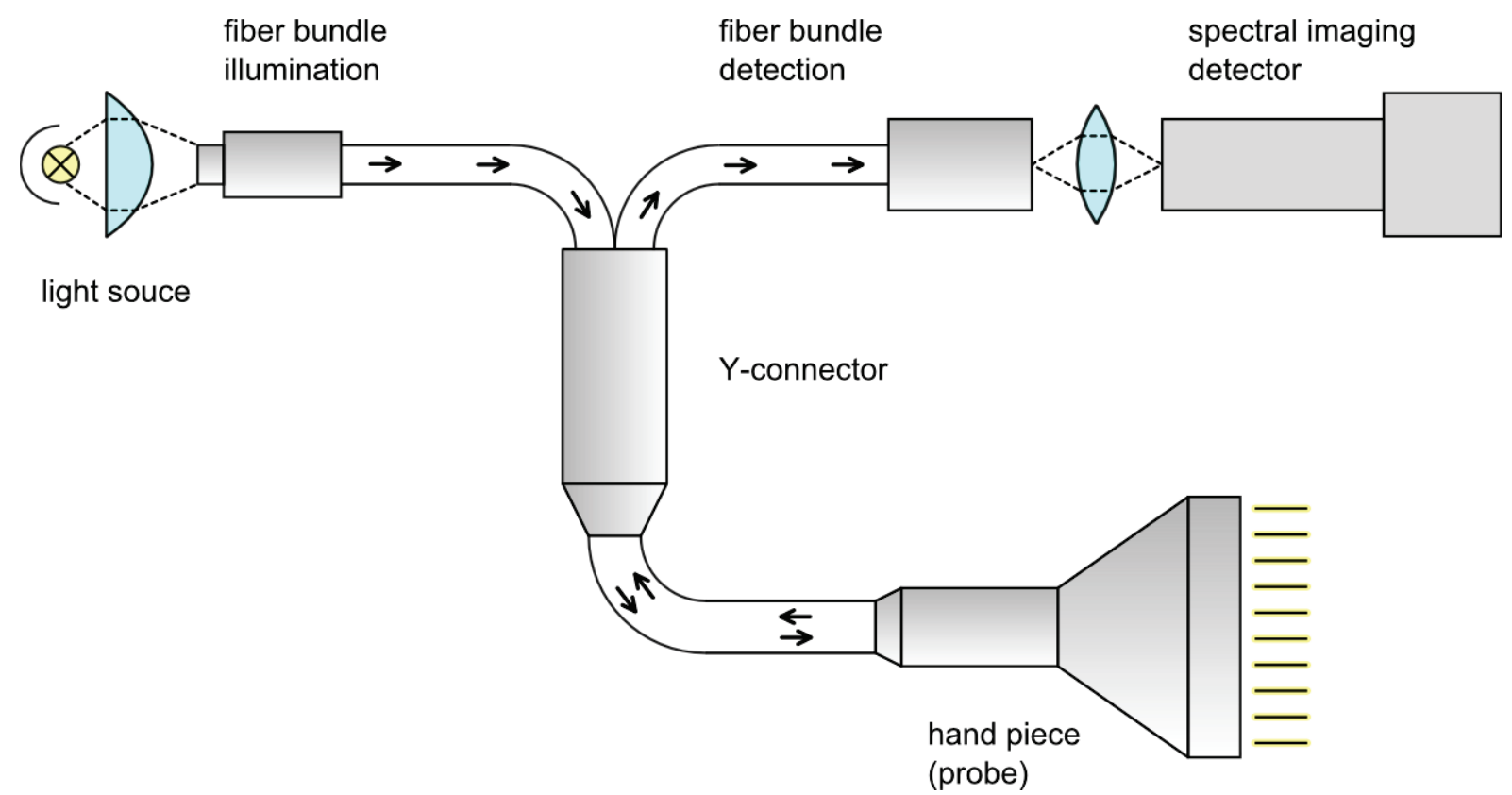

Fig. 3, Structure of the skin probe consisting of two optical branches for illumination and detection 
A specially developed hand piece holds the illuminating fibers at the other end. The skin is illuminated through these fibers. At each end of a fiber, the emerging light is collimated by a micro lens (Fig. 4). The sensors hand piece has a matrix of micro lenses, one for each of the 144 illuminating fibers. The micro lenses are arranged in a grid of $40 \times 40 \mathrm{~mm}^{2}$ at a distance of $5 \mathrm{~mm}$ (Fig. 5). Thus the skin is illuminated by de facto parallelized light, which allows for image capture largely independent of the distance of the probe to the skin within a range of up to $10 \mathrm{~mm}$. Optical fibers for detection are arranged between the illuminating fibers. They also have collimating lenses. They gather the remission from the upper layers of the skin. The collection of surface reflected light is avoided to a large extent by the fact that the illuminating and detecting fibers are arranged in a chessboard-like pattern in the hand piece.

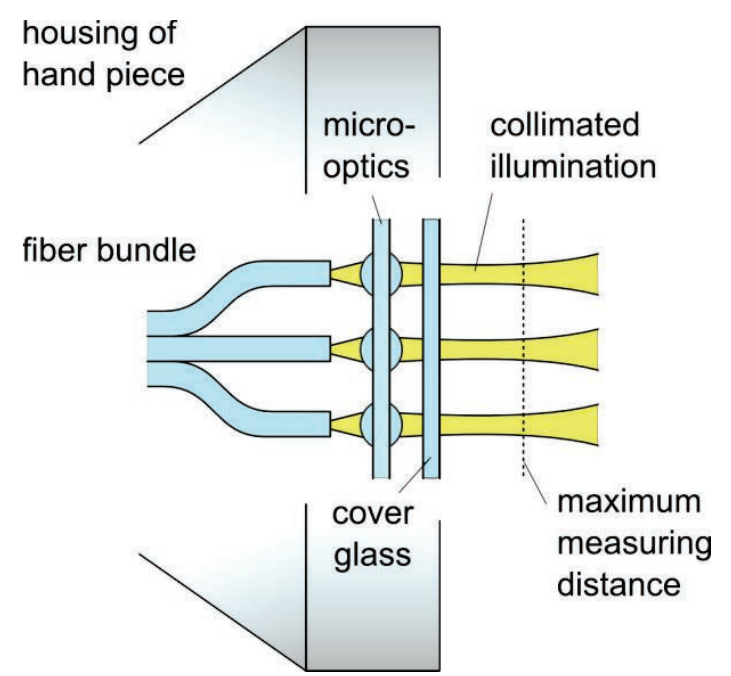

Fig. 4, Internal design of the hand piece comprising fiber bundle and micro lens array

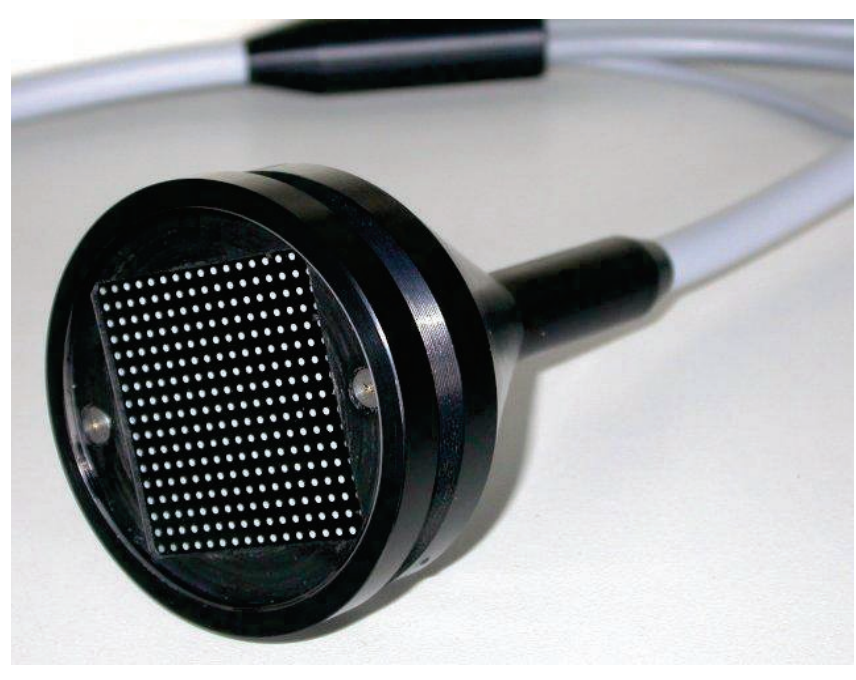

Fig. 5, Hand piece of the spectroscopic skin probe with an array of micro lenses for distance-independent image recording

Altogether, 109 fibers in the hand piece are transferring the emission from the skin to the imaging spectrograph. Within a coupling module, the individual fibers are arranged linearly and projected through a reducing optical system on the entrance slit of the spectrograph. Latter spectrally disperses the light from the sample and projects it on a camera chip. The resulting image contains the full spectral and spatial information about the wound.

\section{Processing hyperspectral data of wounds}

Algorithms were developed to process and convert the 2-D-hyperspectral image (Fig. 6) to an image format that can be intuitively interpreted and appreciated by medical doctors. The influence of ambient light can be compensated for by fast successive measurements with and without integrated probe lighting. The remission from each of the 109 detecting fibers illuminates several pixels on the detector chip producing multiple spectra per fiber. After removing the disturbing ambient light effects from the acquired hyperspectral image, an algorithm detects the pixels illuminated by each fiber, whereby averaging them to a single corresponding spectrum per fiber leading to a higher signal-to-noise ratio. The remission spectra are then rearranged in a 3-D-hyperspectral cube $(x-y-\lambda)$ with two spatial and a wavelength axis, which is a common format for hyperspectral images.

Generally, remission is represented as a value relative to the maximum expected light signal (scattered back) from the medium in question. Polytetrafluoroethylene (PTFE) shows a characteristic behavior of letting light penetrate the surface and scattering it back as real skin does. Hence, a one centimeter thick block of PTFE can be used as a reference white standard. The remission is then calculated as stated in F.1.

$$
\text { remission in } \%=\frac{\text { spectral image }- \text { ambient image }}{\text { white standard }- \text { dark current }} \times 100
$$




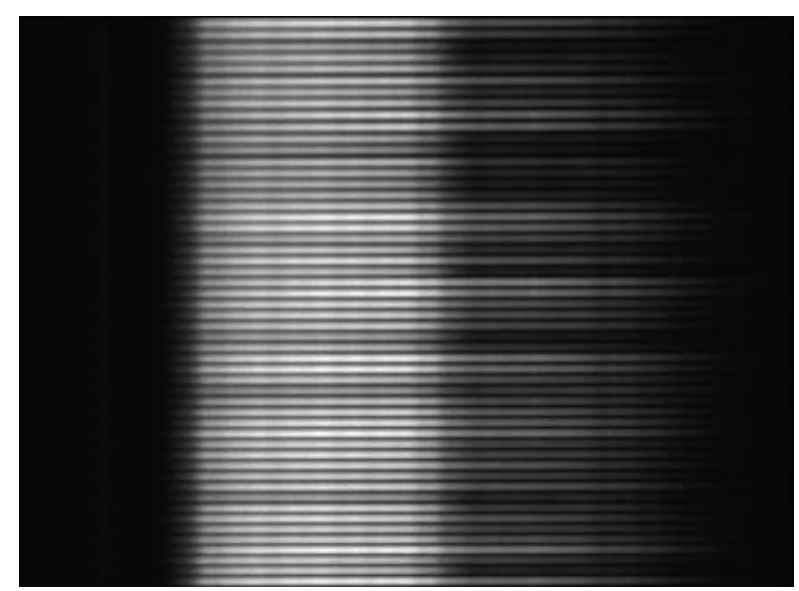

Fig. 6, Detail of a spectral image of a chronic wound showing single fiber channels with different spectral responses of the skins remission

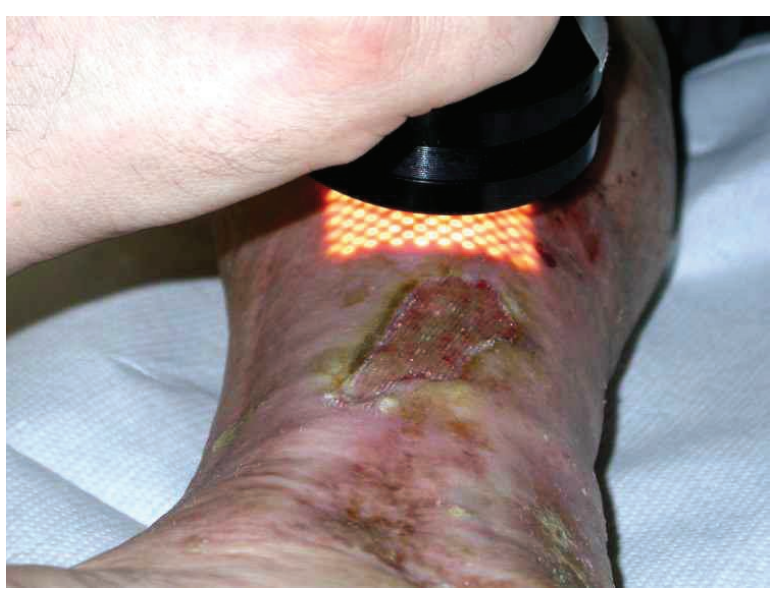

Fig. 7, Measurement of a chronic wound at the clinical survey for gathering data for the spectral library

Several measurements were carried out on wounds from patients at a hospital. An experienced physician examined and classified each wound based on the aforementioned Wollina Scale. Multivariate data analysis methods are applied to the data, whereby all data is loaded in to a single augmented data set in the mathematical software environment MATLAB. A combination of principal component analysis (PCA) and Kaiser VARIMAX [6], Orthogonal Projection Approach (OPA) [7], as well as Multivariate Curve Resolution (MCR) [8] is used to construct a spectral library of characteristic remission spectra correlated to the Wollina wound score.

While PCANARIMAX is useful to estimate the number of most significant components in the dataset, the associated spectra can be extracted using OPA. By associating the selected spectra to the classified wounds, groups of spectra belonging to a score are constructed. However, since the physician made an integral judgment for heterogeneous wounds, the direct mapping of spatially resolved spectra leads to groups with intersecting boundaries. By employing thresholds and also manually comparing the spectra to photographs of the wounds, the choice of significant spectra can be narrowed down to three groups. A group representing scores $2 \& 3$, another scores $4 \& 5$ and the last scores $6 \& 7$. Bearing in mind, that in practice the results should be simple and comprehensible, but yet contain as much information as possible for the physician, three to four classes should be a good scale. Unfortunately there was not enough data to associate with the score group 0 \& 1 . The spectra in a score group were averaged to make a spectral library comprising three artificial remission spectra that are used to classify subsequent measurements.

Component analysis or other classification methods can be applied to the spectral data. For instance, a score is computed that describes the state of a wound numerically. MCR and Spectral Angle Mapping (SAM) were used to classify the wound data. MCR is a component analysis algorithm that can be used on data described by a linear model. It performs well as a tool for quasi unsupervised data-mining, whereby its strength lies in the use of constraints, in which case the fitting process can be controlled on the basis of a priori knowledge. With this method, latent information buried in the data can be discovered. MCR would reproduce the optimized (optional) input spectra and so called concentration maps, which show the spatial distribution of each member of the spectral library.

As a classifier MCR showed a poor performance, due to the covariance present in the data, whereas SAM gave better results. SAM compares unknown spectra to one or more known spectra and can, therefore, make an assignment to a class depending upon similarity. The measure for similarity is the angle between the reference and the spectrum to be classified. Here, the spectrum is considered to be made of vectors spanning an $n$-dimensional vector space from the point of origin in the coordinate system and $n$ equals the number of spectral channels. Each vector has a defined position and direction in the coordinate system, so an angle is formed between the each vector of the reference spectrum (known object class) and the unknown spectrum. The angle a corresponds to the scalar or dot product of the vectors in question (F.2).

$$
\propto=\cos ^{-1}\left(\frac{\sum_{i=1}^{n} t_{i} r_{i}}{\left(\sum_{i=1}^{n} t_{i}^{2}\right)^{\frac{1}{2}}\left(\sum_{i=1}^{n} r_{i}^{2}\right)^{\frac{1}{2}}}\right)
$$


A small angle between the vectors depicts a high mark for similarity and vice versa. In the training phase an appropriate threshold for each score group was determined empirically. The SAM method considers only the position and direction of the vectors and not the length, which encodes the amplitude of the spectrum. Hence variance in the luminance is ignored by the method, making it robust and highly sensitive to differences in the form of the spectra.

The determination of the wound condition is done by measuring and comparing the wound spectra with the library of spectral signatures. To generate this library, measurements were carried out on 16 chronic wounds (Fig. 7). For the purpose of calibration, an experienced physician determined a wound specific value on the eight-level scale using a set of criteria according to Wollina.

\section{Laboratory and clinical study of the skin probe}

The skin probe should be able to evaluate closely neighboring areas of the wound separately. Therefore, the spatial resolution was examined, with the help of simulated structures in the form of coated plastic foils. On the initially transparent foils, bars with different periodicity were printed and used as a skin model in transmission for image recording with the skin probe. A clear reproduction of structures with a periodicity of one line pair per centimeter could be demonstrated. The investigation showed that the resolution was sufficient for the quantitative evaluation of the extent of chronic wounds and their components.

Repetitive measurements resulted in a reproducibility of $0.8 \%$ (RSD) on use of the flat PTFE reference sample and 30-fold application of the probe on the sample, at a distance of $7.5 \mathrm{~mm}$. Hence, a sufficient stability of the sensor is proven under ideal conditions. In the case of measurements on humans at a distance between $5 \mathrm{~mm}$ and $10 \mathrm{~mm}$ to the skin and poor lighting conditions, a reproducibility of $2.2 \%$ (RSD) was determined. For this experiment, healthy skin of the palm was also illuminated with a fluorescent lamp, in order to test the correction function of the skin probe against ambient light. Here again, 30 consecutive measurements were made, whereby the hand piece was newly positioned each time on the measured surface.

In a clinical survey 16 wounds were measured with the skin probe, whose data are also used for the spectral library. With this data the objective determination of wound scores could be demonstrated. The evaluation of spectral data of chronic wounds taken with the probe resulted in an agreement with the judgment of a physician in $69 \%$ of the cases. Whereby, a deviation of the calculated wound score of 0.5 points was rated as a match. For example, the ulcus in Fig. 8 was estimated by the physician to have a wound score of 3.0, while the skin probe computed 3.5 using MCR and SAM as evaluation methods. The wound score images indicate the agreement of a wound area with a wound score by their brightness (high / low).

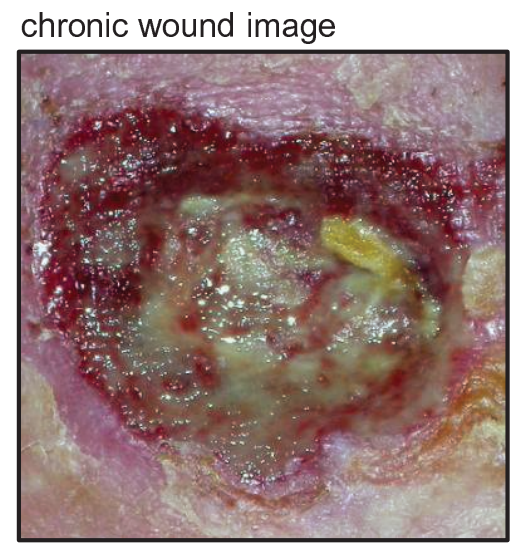

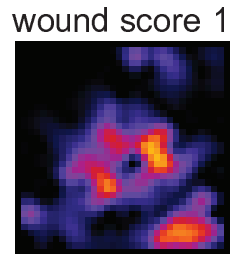
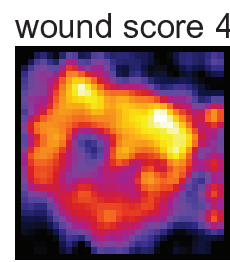

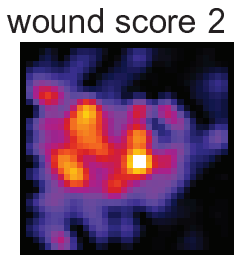

wound score 5

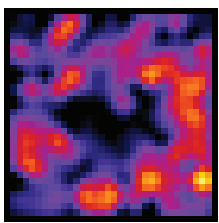

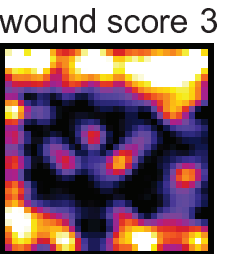

wound score 6

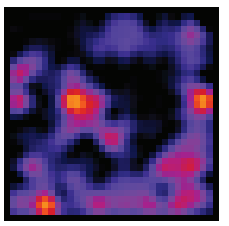

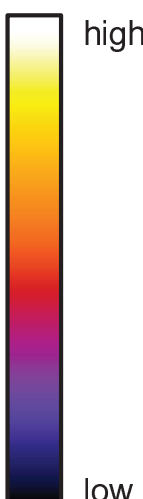

low

Fig. 8, Digital image of a chronic wound (ulcus cruris venosum) on the foot of a patient (left), wound score images from the skin probe (right), classification by the MCR method

As repetitive measurements with respect to reproducibility have shown, the skin probe makes an objective quantitative comparison of wound remission for an extended period of time possible. This establishes an important prerequisite to support future medical studies during the typical healing time of chronic wounds. With these results not only overall statements about the healing prospects of the wound can be made, but also certain parts of the wound can be evaluated.

In addition to the currently used method of remission measurement with a halogen light source, the probe can be used in principle for skin examination by fluorescence spectroscopy and Raman spectroscopy. 
This would require the adaptation of components of the system to the respective excitation and detection method. The principle of imaging spectroscopy however remains the same. In this context, plans for studies as part of advancements in the development of the skin sensor are in progress.

Furthermore, the spectral imaging sensor can be used for applications outside the field of medicine. Whether in the industrial material testing or quality control in the food industry, the skin imaging probe provides a universal tool for spectroscopic evaluation of all biological and non-biological surfaces.

This work is funded by the BMBF (Project No. 0330463C).

\section{Literature}

[1] F. Lang, H. Lippert, S. Piatek, W. Vanscheidt, H. Winter: Häufige Probleme bei der Behandlung chronischer Wunden. HARTMANN WundForum, 1/1996, S.27.

[2] W.D. Schmidt, K. Liebold, D. Fassler, U. Wollina. Contact-free spectroscopy of leg ulcers: principle, technique, and calculation of spectroscopic wound scores. J. Invest. Dermatol., 2001, Vol.116(4), pp. $531-5$.

[3] W.D. Schmidt, A. Scheibe, D. Faßler: Anordnung für einen Messkopf zur kombinierten Aufnahme von Remissions- und Fluoreszenz-Spektren sowie von Bildern, vorzugsweise zur Anwendung in der Dermatologie. Patent DE102005036147, Erteilung 10.04.2008.

[4] F. Erfurth, A. Tretyakov, B. Nyuyki, G. Mrotzek, W.D. Schmidt, D. Fassler, H.P. Saluz: Two-laser, large field hyperspectral microarray scanner for the analysis of multicolour microarrays. Analytical Chemistry, 2008, Vol.80(20), pp. 7706-13.

[5] R.Knetsch; W.Arnold; F.Erfurth; A.Scheibe; B.Nyuyki, W.D.Schmidt, Bildgebende optische Spektralsensorik zur automatischen Regelung von Gasbrennern für die Glas verarbeitende Industrie. VDI-Tagungsband: 24. Deutscher Flammentag - Verbrennung und Feuerung, 2009, S.251-6.

[6] H.F. Kaiser. The varimax criterion for analytic rotation in factor analysis. Psychometrika, 1958, Vol.23, pp. 187-200.

[7] F.C. Sanchez, J. Toft, B. van den Bogaert, D.L. Massart. Orthogonal Projection Approach Applied to Peak Purity Assessment. Analytical Chemistry, 1996, Vol.68(1), pp. 79-85.

[8] R. Tauler. "MCR-ALS", Group of Solution Equilibria and Chemometrics, http://www.mcrals.info/, Chemical and Environmental Research Institute of Barcelona (IIQAB), University of Barcelona, 2006. 